\title{
Philosophy, understanding and the consultation: a fusion of horizons
}

Early models of the consultation were 'disease' centred with little consideration of the patients perspective. Later models advised we should gain the 'patients understanding' or better 'shared understanding' as used by Pendleton, Schofield, Tate, and Havelock. ${ }^{1}$ The profession is now developing the idea of patient-centred medicine; it is gaining popularity but still practised infrequently even when looked for in the MRCGP video examination. ${ }^{2}$ For the consultation process to be successful the patient needs to feel heard and understood. Philosophical thought can offer ideas to describe this task of understanding.

The works of the German philosopher Hans-Georg Gadamer (1900-2002) explain that 'the modern concepts of science are not adequate to understand people and our experience of art and even communication'. ${ }^{3}$ He developed a philosophical perspective in his work 'Truth and Method' and explained a process of philosophical hermeneutics.

Hermeneutics is a process which helps interpretation and understanding things from someone else's perspective. It can be applied to situations where we encounter meanings that are not easily understood but require some effort to interpret. He originally applied this to an interpreter and a religious text but in a later essay he describes 'its (hermeneutics) fundamental significance for our entire understanding of the world and thus for all the various forms in which this understanding manifests itself: from interhuman communication to manipulation of society'. ${ }^{4}$

A recent example of applying this hermeneutic process to human processes was to understand 'hope' in a teenage population, which on first hearing sounds an impossible task. Gadamer's hermeneutics provides insight into this very human process and an interesting research method. ${ }^{5}$ I would like to suggest that this process could be applied to a consultation between patient and doctor.
When applying hermeneutics to the human process of interpretation Gadamer talks of a 'horizon' as a way to conceptualise understanding. Your horizon is as far as you can see or understand. Both patient and doctor go into a consultation with a horizon and out of this encounter both will leave with their own new horizon. Gadamer describes a horizon as 'The totality of all that can be realised or thought about by a person at a given time in history and in a particular culture.'

Gadamer states that: 'the concept of horizon suggests itself because it expresses the superior breadth of vision that the person who is trying to understand must have. To acquire a horizon means that one learns to look beyond what is close at hand - not in order to look away from it but to see it better'. 3

Understanding happens when our present understanding or horizon is moved to a new understanding or horizon by an encounter. $^{3}$ Thus the process of understanding is a 'fusion of horizons'. The old and the new horizon combining into something of living value.

We could see the 'fusion of horizons' as a metaphor for the consultation process, a simple image of what happens in a consultation, with patient and doctor interacting and resulting in changed horizons.

There are several ideas to explore on the way to developing our horizon as detailed by Gadamer. These are: pre-understanding, prejudices, fore-conceptions, 'Bildung' or openness to meaning, language, and imagination. Both the patient and doctor will be running these processes. Both enter and leave the consultation with separate horizons and will have undergone a fusion of horizons.

\section{Pre-understanding}

As we enter an encounter we already have preformed ideas. Essentially, we have a history and an understanding of the world before we begin to think about it. ${ }^{6}$

\section{Prejudices}

We all take ideas and attitudes into a situation. Gadamer calls these 'prejudices' not in our negative sense, but: 'A judgement that is rendered before all elements that determine a situation have been finally examined. ${ }^{3}$ For instance, a patient may have already decided they are not worried about their blood pressure or that if the doctor doesn't examine their chest the examination will be inadequate.

\section{Fore-meanings}

During an encounter we are always assuming it has meaning and completeness unless it is completely unintelligible. We are constantly trying to look ahead to find a meaningful understanding. This happens before we settle on our final view. A patient may ask for the pill which we think is a straight forward request but it may open out into a dialogue about infertility which was the main topic on the patient's agenda. Gadamer states we cannot stick blindly to our own fore-meaning if we want to understand the meaning of another and goes on to state that 'All that is asked is that we remain open to the meaning of the other person'. ${ }^{3}$ This leads us to the next stage of understanding.

\section{'Bildung' or openness to meaning}

The roots of Bildung go back to medieval mysticism and Bildung translates from the German to 'education'. However, there is a much wider definition in German involving 'form', 'image' and 'picture'. For the purpose of application to an encounter it means we must be open to reforming, open to meaning and change. With Bildung one moves from the all-too-familiar and learns to allow for what is different from oneself. This process is vital if we are to gain insight into a patient's concerns and help them to change their horizon. We must be open to their understanding.

\section{A fusion of horizons}

'Understanding' is the fusion of our past and 
present horizon. Indeed, the present cannot be formed without the past. Past and present cannot exist without each other and 'understanding is always the fusion of these horizons supposedly existing by themselves.'

\section{Language}

What is the meaning of 'understanding'? What is the meaning of meaning? One of the most important aspects here is our language. The power of language was expressed by Wittgenstein: 'The limits of my language mean the limits of my world'. Gadamer also says that 'understanding itself has a fundamental connection with language'. ${ }^{3}$ This can also be expressed as 'Language and understanding of transmitted meaning are not two processes but one and the same. ${ }^{77}$ The language of the doctor and that of the patient are therefore crucial to understanding. At extremes this is obvious but we all get blank looks after explanations using medical terminology and I still have to work hard to understand the language of philosophy! An appreciation of our language and that of the patient is required if we are to facilitate understanding.

\section{Imagination}

Perhaps a rather surprising idea during the busy surgery! The scope of imagination is described by Einstein: 'logic can take you from A to B, but imagination can take you everywhere.' Gadamer points out, 'the differentia between methodological sterility and genuine understanding is imagination, that is, the capacity to see what is questionable in the subject matter and to formulate questions that question the subject matter further." ${ }^{\prime}$ Others have produced tools such as 'Socratic Questioning' to apply this power but without the underlying imagination it may lead simply from A to B! Using creative ideas and application we may find another view for the patient who is stuck and unable to see a way forward. This question came to me when seeing a patient who was bereaved, 'could you ever imagine a day when you will wake up and be able to think of the wonderful things they gave to you?' At the right time this might help a change of perspective.

\section{Circle of understanding}

'The movement of understanding is constantly from the whole to part and back to the whole'. ${ }^{3}$ We are constantly breaking apart our understanding comparing it to another view or new experience and then putting it back together to produce a new understanding or horizon. During this process we apply our pre-understanding, involve our prejudices, and make use of our fore-conceptions, language and imagination. All the time we remain open to meaning. We need this circular movement because 'nothing that needs interpretation can be understood at once.'

This view of a consultation describes separate horizons, the doctor's and the patient's. Both will leave with a new horizon, the old horizon will be changed by the encounter and a new one appears (Figure 1). However, as Gadamer explains we do not move into a new horizon, our horizon moves with us. The narrative model of the consultation ${ }^{9}$ would agree with this. We listen to their story and help them to add a little more, we don't simply wipe out their story and write a new one.

For Gadamer, the doctors encounter with the patient can enable them to see new horizons. We cannot simply drop in a new story or horizon. We need to help them to a new understanding and always remember that the patients' horizon and ours are different.

From the doctors perspective our horizons may change after each consultation and I like to think of this as gaining wisdom from our patients. As a GP my consultations are fine-tuned by encounters, both for individual patients and between patients with similar backgrounds and problems. The doctor's horizon can be changed after each consultation. Our wisdom is increased as experience grows. On an individual basis the doctors understanding of a patient's horizon is changed, we could even look back on a consultation after a surgery and form another horizon. Problem case analysis is an excellent way to facilitate this. At the start of the next encounter we start with a different horizon, it is never finished, final or the 'ultimate truth'. We are constantly forming a new understanding, fusing our horizons.

A patient may leave the surgery without the view the doctor may want, or the doctor may not have a clear picture of why the patient attended. Our history and background must play a part in forming our present horizon and so contribute to our new one. We can only offer explanations and images to use in the formation of a new view and then the final horizon is the individuals alone. This means that 'Understanding is not reconstruction but mediation'. ${ }^{8}$ Therefore, we cannot dispose of the old meaning and start again; we must use it in a negotiation to arrive at a new understanding.

Gadamer's insights show us that there are two hermeneutic processes happening in a consultation, that of the patient and the doctor. Each has pre-understanding, foreconceptions and so on. The encounter is complicated because the doctor and patient have different consultation styles. John Launer describes two consultation styles in 'uniqueness and conformity'. ${ }^{9}$ Here he talks of a patients 'narrative style' whereby they need to tell their story and the doctors 'normative style' which involves pattern recognition (does this fit with angina?). Philosophical hermeneutics explains why this happens; the patient and doctor come from different backgrounds and both bring their own ideas and processes to the encounter.

Figure 1. Circles of understanding in a consultation



Patient's circle of understanding

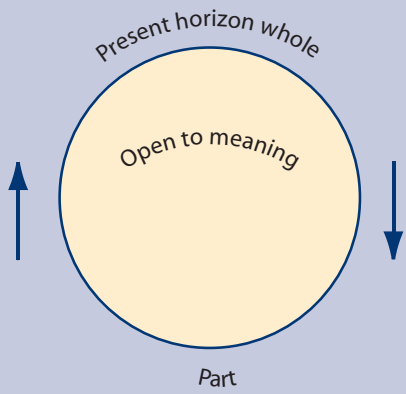

GP's circle of understanding 
As Launer explains when managing each consultation it 'goes far beyond so-called 'patient-centred' medicine. It means recognising the equal legitimacy of the patient's need for self-expression and one's own need as a doctor to achieve pattern recognition, action and closure. ${ }^{10}$

Both patient and doctor need to go around their circle of understanding. Acknowledging these two circles of understanding in conversation could help the doctor to appreciate the skill of using careful listening, questioning and imaginative ideas to alter a patient's horizon. A skill which I feel is the very essence of general practice.

I offer these ideas as a way to appreciate what is happening when we have a dialogue with a patient. Helping the patient to 'finetune' their understanding is crucial in a good consultation as this enables them to change their horizon. For the doctor there may be other benefits. Gadamer says that 'the real power of hermeneutical consciousness is our ability to see what is questionable'. ${ }^{6}$ It enables us to join our experience of science to our own universal and human experience of life. It is during this process that I also believe the doctor gains wisdom.

\section{Jeff Clark}

\section{REFERENCES}

1. Pendleton D, Schofield T, Tate P, Havelock P. The consultation: an approach to learning and teaching. Oxford: Open University Press, 1984.

2. Campion P, Foulkes J, Neighbour R, Tate P. Patient centredness in the MRCGP video examination: analysis of large cohort. BMJ 2002; 325: 691-692.

3. Gadamer H-G. Truth and method. 2nd revised edn. London: Continuum International Publishing Group, 2004.

4. Gadamer H-G. On the scope and function of hermeneutical reflection (1967). In: Linge DF Philosophical hermeneutics. Berkeley, CA: University of California Press, 1977: 18-43.

5. Turner, d S. Horizons revealed: From methodology to method. International Journal of Qualitative Methods 2003, 2(1): Article 1. http://www.ualberta.ca/ iiqm/ backissues/2_1/html/turner.html (accessed 27 Nov 2007).

6. Gadamer H-G. The universality of the hermeneutical problem (1966). In: Linge DE. Philosophical hermeneutics. Berkeley, CA: University of California Press, 1977: 3-17.

7. Wittgenstein L. Tractatus Logico-Philosophicus. London: Kegan Paul, Trench, Trubner, 1922.

8. Linge DE. Philosophical hermeneutics. Berkeley, CA: University of California Press, 1977.

9. Launer J. Narrative-based primary care: a practical guide. Abingdon: Radcliffe, 2002.

10. Launer J. Uniqueness and conformity. Q J Med 2003; 96: 615-616.

DOI: 10.3399/bjgp08X263929

\section{Salaried doctors: the problems}

In general practice differing groups face different problems. The relative professional isolation of salaried GPs employed by practices leaves them vulnerable to exploitation and support mechanisms for them are often 'ad hoc' or non-existent. Satisfaction in a salaried post is associated with a robust and fair contract, interest from employers in the skills and attributes of the GP, and a commitment to career development. In some situations these are lacking for the salaried GP, leading to de-motivation and a search for employment in other areas of the health service. In my work as an appraiser, trainer, and GP for 30 years I have increasingly recognised this as a problem.

One salaried GP described to me her low self-esteem and loss of selfconfidence following years in a practice where she rarely met the partners and was never asked to practice meetings. Another felt overwhelmed by the demands of patients who preferred her to the other partners. As a conscientious, caring, and careful GP, inevitably her surgeries would overrun and this was compounded by 'hot bedding', which often meant that she could not gain access to a consulting room in time to start her surgery. With childcare arrangements and school collecting times she was always stressed and pulled in different directions. Attempts to modify her contract fell on deaf ears and now she is considering a career outside general practice.

Other GPs have equally harrowing tales. Another of bullying by partners in a practice where one partner sat with his door open listening to her surgeries and interrogating any of her patients on leaving to see if they were 'upset'. As a caring doctor, her consultation rate for depression was high and of course many patients were distressed. However, they were very appreciative of her skills. She was able to confront the partner's behaviour but at great cost to her selfesteem and confidence.
A practice told another GP who had worked conscientiously for 2 years that his services were no longer required with no explanation and with 1 month's notice. $\mathrm{He}$ had never had an appraisal in the practice and no partner had ever told him of any problems. He was informed of the termination of his employment by the practice manager.

Logistics are another hurdle in the life of a salaried doctor. If they move around several practices no room is the same. They often resort to carrying their own equipment and forms as it saves time. Local protocols and clinical pathways might pass them by as they are out of the information loop.

Safeguarding good doctors from exploitation is imperative if general practice is to continue to fulfil the needs of the future. Whatever we may feel about salaried practice, it is undoubtedly here to stay. A salaried GP costs a practice $£ 7000$ to replace and makes retention a priority. It is time to listen to the voices from this group and give them the recognition they deserve.

\section{Julia Lecky}

\section{FURTHER READING}

Aquino P, Kohli B. Salaried general practitioners. BMJ Career Focus 2002; 325: S89a

BMA Salaried GP Contracts: http://www.bma.org.uk/ap.nsf/Content/Hubsalariedgps

DOI: 10.3399/bjgp08X263938 